\title{
The Cocycle of the Quantum HJ Equation and the Stress Tensor of CFT
}

\author{
Marco Matone \\ Department of Physics "G. Galilei" - Istituto Nazionale di Fisica Nucleare \\ University of Padova, Via Marzolo, 8 - 35131 Padova, Italy
}

Received on 15 February, 2005

\begin{abstract}
We consider two theorems formulated in the derivation of the Quantum Hamilton-Jacobi Equation from the EP. The first one concerns the proof that the cocycle condition uniquely defines the Schwarzian derivative. This is equivalent to show that the infinitesimal variation of the stress tensor "exponentiates" to the Schwarzian derivative. The cocycle condition naturally defines the higher dimensional version of the Schwarzian derivative suggesting a role in the transformation properties of the stress tensor in higher dimensional CFT. The other theorem shows that energy quantization is a direct consequence of the existence of the quantum HamiltonJacobi equation under duality transformations as implied by the EP.
\end{abstract}

\section{HJ EQUATION AND COORDINATE TRANSFORMATIONS}

Let us consider the Hamilton-Jacobi (HJ) equation for a one dimensional system. This is obtained by considering the canonical transformation $(q, p) \rightarrow(Q, P)$ leading to a vanishing Hamiltonian

$$
\tilde{H}=0 \text {. }
$$

The old and new momenta are expressed in terms of the generating function of such a transformation, the Hamilton's principal function

$$
p=\frac{\partial \mathcal{S}^{c l}}{\partial q}, \quad P=c n s t=-\left.\frac{\partial \mathcal{S}^{c l}}{\partial Q}\right|_{Q=c n s t}
$$

that satisfies the classical $\mathrm{HJ}$ equation

$$
H\left(q, p=\frac{\partial \mathcal{S}^{c l}}{\partial q}, t\right)+\frac{\partial \mathcal{S}^{c l}}{\partial t}=0
$$

In the case of a time independent potential the time dependence in the Hamilton's principal function $\mathcal{S}^{c l}$ is linear, that is

$$
\mathcal{S}^{c l}(q, Q, t)=\mathcal{S}_{0}^{c l}(q, Q)-E t
$$

where $E$ denotes the energy of the stationary state. It follows that $\mathcal{S}_{0}^{c l}$, called Hamilton's characteristic function or reduced action, satisfies the Classical Stationary HJ Equation (CSHJE)

$$
H\left(q, p=\frac{\partial \mathcal{S}_{0}^{c l}}{\partial q}\right)-E=0,
$$

that is $(\mathcal{W}(q) \equiv V(q)-E)$

$$
\frac{1}{2 m}\left(\frac{\partial \mathcal{S}_{0}^{c l}}{\partial q}\right)^{2}+\mathcal{W}=0 .
$$

Following [1] we now consider a similar question to that leading to the CSHJE but starting with

$$
p=\frac{\partial \mathcal{S}_{0}^{c l}}{\partial q}
$$

rather than with $p$ and $q$ considered as independent variables. More precisely, given a one dimensional system, with time-independent potential (the higher dimensional timedependent case is considered in [2]) we look for the coordinate transformation $q \rightarrow q_{0}$ such that

$$
\mathcal{S}_{0}^{c l}(q) \quad \stackrel{\text { Coord.Transf. }}{\longleftrightarrow} \quad \tilde{\mathcal{S}}_{0}^{c l 0}\left(q_{0}\right),
$$

with $\tilde{\mathcal{S}}_{0}^{c l 0}\left(q_{0}\right)$ denoting the reduced action of the system with vanishing Hamiltonian. Note that in (1) we required that this transformation be an invertible one. This is important point since by compositions of the maps it follows that if for each system there is a coordinate transformation leading to the trivial state, then even two arbitrary systems are equivalent under coordinate transformations. Imposing this apparently harmless analogy immediately leads to rather peculiar properties of Classical Mechanics (CM). First, it is clear that such an equivalence principle cannot be satisfied in $\mathrm{CM}$, in other words given two arbitrary systems $a$ and $b$, the condition

$$
\mathcal{S}_{0}^{c l b}\left(q_{b}\right)=\mathcal{S}_{0}^{c l a}\left(q_{a}\right),
$$

cannot be generally satisfied. In particular, since

$$
\tilde{\mathcal{S}}_{0}^{c l 0}\left(q_{0}\right)=\text { cnst }
$$

it is clear that (1) is a degenerate transformation. However, in principle, by itself the failure of (2) for arbitrary systems would be a possible natural property. Nevertheless, a more careful analysis shows that such a failure is strictly dependent on the choice of the reference frame. This is immediately seen by considering two free particles of mass $m_{a}$ and $m_{b}$ moving with relative velocity $v$. For an observer at rest with respect to the particle $a$ the two reduced actions are

$$
\mathcal{S}_{0}^{c l a}\left(q_{a}\right)=\mathrm{cnst}, \quad \mathcal{S}_{0}^{c l b}\left(q_{b}\right)=m_{b} v q_{b} .
$$

It is clear that there is no way to have an equivalence under coordinate transformations by setting $\mathcal{S}_{0}^{c l b}\left(q_{b}\right)=S_{0}^{c l a}\left(q_{a}\right)$. This means that at the level of the reduced action there is no coordinate transformation making the two systems equivalent. However, note that this coordinate transformation exists if we consider the same problem described by an observer in a frame in which both particles have a non-vanishing velocity so that the two particles are described by non-constant reduced actions. 
Therefore, in CM, it is possible to connect different systems by a coordinate transformation except in the case in which one of the systems is described by a constant reduced action. This means that in CM equivalence under coordinate transformations is frame dependent. In particular, in the CSHJE description there is a distinguished frame. This seems peculiar as on general grounds what is equivalent under coordinate transformations in all frames should remain so even in the one at rest.

\section{THE EQUIVALENCE POSTULATE}

The above investigation already suggests that the concept of point particle itself cannot be consistent with the equivalence under coordinate transformations. In particular, it suggests that the system where a particle is at rest does not exist at all. If this would be the case, then the above critical situation would not occur simply because the reduced action is never a constant. This should reflect in two main features. First the classical concept of point particle should be reconsidered, secondly the CSHJE should be modified accordingly. A natural suggestion would be to consider particles as a kind of string with a lower bound on the vibrating modes in such a way that there is no way to define a system where the particle is at rest. It should be observed that this kind of string may differ from the standard one, rather its nature may be related to the fact that in general relativity is impossible to define the concept of relative stability of a system of particles.

We now start imposing the equivalence under coordinate transformations. The key point is to consider, like in general relativity, the (analogous of the) reduced action as a scalar field under coordinate transformations.

We postulate that for any pair of one-particle states there exists a field $\mathcal{S}_{0}$ such that

$$
\mathcal{S}_{0}^{b}\left(q_{b}\right)=\mathcal{S}_{0}^{a}\left(q_{a}\right),
$$

is well defined. We also require that in a suitable limit $\mathcal{S}_{0}$ reduces to $\mathcal{S}_{0}^{c l}$. Eq.(3) can be considered as the scalar hypothesis. Since the conjugate momentum is defined by

$$
p_{i}=\frac{\partial}{\partial q^{i}} \mathcal{S}_{0}(q)
$$

it follows by (3) that the conjugate momenta $p^{a}$ and $p^{b}$ are related by a coordinate transformation

$$
p_{i}^{b}=\Lambda_{i}^{j} p_{j}^{a}
$$

where $\Lambda_{i}^{j}=\partial q_{a}^{j} / \partial q_{b}^{i}$. Note that we have the invariant

$$
p_{i}^{b} d q_{b}^{i}=p_{i}^{a} d q_{a}^{i} .
$$

Since (3) holds for any pair of one-particle states, we have $\operatorname{Det} \Lambda(q) \neq 0, \forall q$.

The scalar hypothesis (3) implies that two one-particle states are always connected by a coordinate transformation, for such a reason we may equivalently consider (3) as imposing an Equivalence Postulate (EP). In particular, while in arbitrary dimension the coordinate transformation is given by imposing (4), in the one dimensional case the scalar hypothesis immediately leads to

$$
q_{b}=S_{0}^{b^{-1}} \circ S_{0}^{a}\left(q_{a}\right) .
$$

We now consider the consequences of the EP (3). Let us denote by $\mathcal{H}$ the space of all possible $\mathcal{W} \equiv V-E$. We also call $v$-transformations the ones leading from a system to another. Eq.(3) is equivalent to require that

For each pair $\mathcal{W}^{a}, \mathcal{W}^{b} \in \mathcal{H}$, there is a $v$-transformation such that

$$
\mathcal{W}^{a}(q) \longrightarrow \mathcal{W}^{a^{v}}\left(q^{v}\right)=\mathcal{W}^{b}\left(q^{v}\right)
$$

This implies that there always exists the trivializing coordinate $q_{0}$ for which $\mathcal{W}(q) \longrightarrow \mathcal{W}^{0}\left(q_{0}\right)$, where

$$
\mathcal{W}^{0}\left(q_{0}\right) \equiv 0
$$

In particular, since the inverse transformation should exist as well, it is clear that the trivializing transformation should be locally invertible. We will also see that since classically $\mathcal{W}^{0}$ is a fixed point, implementation of (6) requires that $\mathcal{W}$ states transform inhomogeneously.

The fact that the EP cannot be consistently implemented in $\mathrm{CM}$ is true in any dimension. To show this let us consider the coordinate transformation induced by the identification

$$
\mathcal{S}_{0}^{c l v}\left(q^{v}\right)=\mathcal{S}_{0}^{c l}(q) .
$$

Then note that the CSHJE

$$
\frac{1}{2 m} \sum_{k=1}^{D}\left(\partial_{q_{k}} \mathcal{S}_{0}^{c l}(q)\right)^{2}+\mathcal{W}(q)=0,
$$

provides a correspondence between $\mathcal{W}$ and $\mathcal{S}_{0}^{c l}$ that we can use to fix, by consistency, the transformation properties of $\mathcal{W}$ induced by that of $\mathcal{S}_{0}^{c l}$. In particular, since $\mathcal{S}_{0}^{c l v}\left(q^{v}\right)$ must satisfy the CSHJE

$$
\frac{1}{2 m} \sum_{k=1}^{D}\left(\partial_{q^{k v}} \mathcal{S}_{0}^{c l v}\left(q^{v}\right)\right)^{2}+\mathcal{W}^{v}\left(q^{v}\right)=0
$$

by (7) we have

$$
\frac{\partial \mathcal{S}_{0}^{c l v}\left(q^{v}\right)}{\partial q^{k v}}=\Lambda_{k}^{i} \frac{\partial \mathcal{S}_{0}^{c l}(q)}{\partial q^{i}}
$$

Let us set $\left(p^{v} \mid p\right)=p^{t} \Lambda^{t} \Lambda p / p^{t} p$. By (8)-(10), we have $\mathcal{W}(q) \longrightarrow \mathcal{W}^{v}\left(q^{v}\right)=\left(p^{v} \mid p\right) \mathcal{W}(q)$, so that

$$
\mathcal{W}^{0}\left(q_{0}\right) \longrightarrow \mathcal{W}^{v}\left(q^{v}\right)=\left(p^{v} \mid p^{0}\right) \mathcal{W}^{0}\left(q_{0}\right)=0 .
$$

Thus we have [1]

$\mathcal{W}$ states transform as quadratic differentials under classical $\nu$-maps. It follows that $\mathcal{W}^{0}$ is a fixed point in $\mathcal{H}$. Equivalently, in $C M$ the space $\mathcal{H}$ cannot be reduced to a point upon 
factorization by the classical v-transformations. Hence, the EP (6) cannot be consistently implemented in CM. This can be seen as the impossibility of implementing covariance of CM under the coordinate transformation defined by (7).

It is therefore clear that in order to implement the EP we have to deform the CSHJE. As we will see, this requirement will determine the equation for $\mathcal{S}_{0}$.

In Ref.[1] the function $\mathcal{T}_{0}(p)$, defined as the Legendre transform of the reduced action, was introduced

$$
\mathcal{T}_{0}(p)=q^{k} p_{k}-\mathcal{S}_{0}(q), \quad \mathcal{S}_{0}(q)=p_{k} q^{k}-\mathcal{T}_{0}(p) .
$$

While $\mathcal{S}_{0}(q)$ is the momentum generating function, its Legendre dual $\mathcal{T}_{0}(p)$ is the coordinate generating function

$$
p_{k}=\frac{\partial \mathcal{S}_{0}}{\partial q_{k}}, \quad q_{k}=\frac{\partial \mathcal{T}_{0}}{\partial p_{k}}
$$

Note that adding a constant to $\mathcal{S}_{0}$ does not change the dynamics. Then, the most general differential equation $\mathcal{S}_{0}$ should satisfy has the structure

$$
\mathcal{F}\left(\nabla \mathcal{S}_{0}, \Delta \mathcal{S}_{0}, \ldots\right)=0
$$

Let us write down Eq.(11) in the general form

$$
\frac{1}{2 m} \sum_{k=1}^{D}\left(\partial_{q^{k}} \mathcal{S}_{0}(q)\right)^{2}+\mathcal{W}(q)+Q(q)=0 \text {. }
$$

The transformation properties of $\mathcal{W}+Q$ under the $v$-maps are determined by the transformed equation

$$
\frac{1}{2 m} \sum_{k=1}^{D}\left(\partial_{q^{k v}} \mathcal{S}_{0}^{v}\left(q^{v}\right)\right)^{2} / 2 m+\mathcal{W}^{v}\left(q^{v}\right)+Q^{v}\left(q^{v}\right)=0,
$$

so that

$$
\mathcal{W}^{v}\left(q^{v}\right)+Q^{v}\left(q^{v}\right)=\left(p^{v} \mid p\right)[\mathcal{W}(q)+Q(q)]
$$

A basic guidance in deriving the differential equation for $\mathcal{S}_{0}$ is that in some limit it should reduce to the CSHJE. In [1][2][3] it was shown that the parameter which selects the classical phase is the Planck constant. Therefore, in determining the structure of the $Q$ term we have to take into account that in the classical limit

$$
\lim _{\hbar \rightarrow 0} Q=0
$$

The only possibility to reach any other state $\mathcal{W}^{v} \neq 0$ starting from $\mathcal{W}^{0}$ is that it transforms with an inhomogeneous term. Namely as $\mathcal{W}^{0} \longrightarrow \mathcal{W}^{v}\left(q^{v}\right) \neq 0$, it follows that for an arbitrary $\mathcal{W}^{a}$ state

$$
\mathcal{W}^{v}\left(q^{v}\right)=\left(p^{v} \mid p^{a}\right) \mathcal{W}^{a}\left(q_{a}\right)+\left(q_{a} ; q^{v}\right),
$$

and by (13)

$$
Q^{v}\left(q^{v}\right)=\left(p^{v} \mid p^{a}\right) Q^{a}\left(q_{a}\right)-\left(q_{a} ; q^{v}\right) .
$$

Let us stress that the purely quantum origin of the inhomogeneous term $\left(q_{a} ; q^{v}\right)$ is particularly transparent once one consider the compatibility between the classical limit (14) and the transformation properties of $Q$ in Eq.(16).

The $\mathcal{W}^{0}$ state plays a special role. Actually, setting $\mathcal{W}^{a}=$ $\mathcal{W}^{0}$ in Eq.(15) yields

$$
\mathcal{W}^{v}\left(q^{v}\right)=\left(q_{0} ; q^{v}\right)
$$

so that, according to the EP (6), all the states correspond to the inhomogeneous part in the transformation of the $\mathcal{W}^{0}$ state induced by some $v$-map.

Let us denote by $a, b, c, \ldots$ different $v$-transformations. Comparing

$$
\mathcal{W}^{b}\left(q_{b}\right)=\left(p^{b} \mid p^{a}\right) \mathcal{W}^{a}\left(q_{a}\right)+\left(q_{a} ; q_{b}\right)=\left(q_{0} ; q_{b}\right),
$$

with the same formula with $q_{a}$ and $q_{b}$ interchanged we have

$$
\left(q_{b} ; q_{a}\right)=-\left(p^{a} \mid p^{b}\right)\left(q_{a} ; q_{b}\right),
$$

in particular $(q ; q)=0$ More generally, imposing the commutative diagram of maps

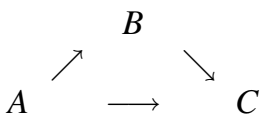

that is comparing

$$
\begin{gathered}
\mathcal{W}^{b}\left(q_{b}\right)=\left(p^{b} \mid p^{c}\right) \mathcal{W}^{c}\left(q_{c}\right)+\left(q_{c} ; q_{b}\right)= \\
\left(p^{b} \mid p^{a}\right) \mathcal{W}^{a}\left(q_{a}\right)+\left(p^{b} \mid p^{c}\right)\left(q_{a} ; q_{c}\right)+\left(q_{c} ; q_{b}\right),
\end{gathered}
$$

with (17) we obtain the basic cocycle condition

$$
\left(q_{a} ; q_{c}\right)=\left(p^{c} \mid p^{b}\right)\left[\left(q_{a} ; q_{b}\right)+\left(q_{b} ; q_{c}\right)\right] \text {, }
$$

which expresses the essence of the EP. In the one dimensional case we have

$$
\left(q_{a} ; q_{c}\right)=\left(\partial_{q_{c}} q_{b}\right)^{2}\left(q_{a} ; q_{b}\right)+\left(q_{b} ; q_{c}\right) .
$$

It is well-known that this is satisfied by the Schwarzian derivative. However, it turns out that it is essentially the unique solution. More precisely, we have [1]

Theorem 1. Eq.(20) defines the Schwarzian derivative up to a multiplicative constant and a coboundary term.

Since the differential equation for $\mathcal{S}_{0}$ should depend only on $\partial_{q}^{k} \mathcal{S}_{0}, k \geq 1$, it follows that the coboundary term must be zero, so that [1]

$$
\left(q_{a} ; q_{b}\right)=-\frac{\beta^{2}}{4 m}\left\{q_{a}, q_{b}\right\}
$$

where $\{f(q), q\}=f^{\prime \prime \prime} / f^{\prime}-3\left(f^{\prime \prime} / f^{\prime}\right)^{2} / 2$ is the Schwarzian derivative and $\beta$ is a nonvanishing constant that we identify 
with $\hbar$. As a consequence, $\mathcal{S}_{0}$ satisfies the Quantum Stationary Hamilton-Jacobi Equation (QSHJE) [1]

$$
\frac{1}{2 m}\left(\frac{\partial \mathcal{S}_{0}(q)}{\partial q}\right)^{2}+V(q)-E+\frac{\hbar^{2}}{4 m}\left\{\mathcal{S}_{0}, q\right\}=0 .
$$

$\psi=\mathcal{S}_{0}^{\prime-1 / 2}\left(A e^{-\frac{i}{\hbar} S_{0}}+B e^{\frac{i}{\hbar} S_{0}}\right)$ solves the Schrödinger Equation (SE)

$$
\left(-\frac{\hbar^{2}}{2 m} \frac{\partial^{2}}{\partial q^{2}}+V\right) \psi=E \psi
$$

The ratio $w=\psi^{D} / \psi$ of two real linearly independent solutions of (22) is, in deep analogy with uniformization theory, the trivializing map transforming any $\mathcal{W}$ to $\mathcal{W}^{0} \equiv 0$ [1][4]. This formulation, proposed in collaboration with Faraggi, extends to higher dimension and to the relativistic case as well [1][2].

Let $q_{-/+}$be the lowest/highest $q$ for which $\mathcal{W}(q)$ changes sign, we have [1]

\section{Theorem 2. If}

$$
V(q)-E \geq \begin{cases}P_{-}^{2}>0, & q<q_{-}, \\ P_{+}^{2}>0, & q>q_{+},\end{cases}
$$

then $w$ is a local self-homeomorphism of $\hat{\mathbb{R}}=\mathbb{R} \cup\{\infty\}$ if and only if Eq.(22) has an $L^{2}(\mathbb{R})$ solution.

The crucial consequence is that since the QSHJE is defined if and only if $w$ is a local self-homeomorphism of $\hat{\mathbb{R}}$, it follows that the QSHJE implies by itself energy quantization. We stress that this result is obtained without any probabilistic interpretation of the wave function.

\section{PROOF OF THEOREM 1}

The main steps in proving theorem 1 are two lemmas [1]. Let us start observing that if the cocycle condition (20) is satisfied by $(f(q) ; q)$, then this is still satisfied by adding a coboundary term

$$
(f(q) ; q) \longrightarrow(f(q) ; q)+\left(\partial_{q} f\right)^{2} G(f(q))-G(q) .
$$

Since $(A q ; q)$ evaluated at $q=0$ is independent of $A$, we have

$$
0=(q ; q)=(q ; q)_{\mid q=0}=(A q ; q)_{\mid q=0} .
$$

Therefore, if both $(f(q) ; q)$ and (24) satisfy (20), then $G(0)=$ 0 , which is the unique condition that $G$ should satisfy. We now use (11) to fix the ambiguity (24). First of all observe that the differential equation we are looking for is

$$
\left(q_{0} ; q\right)=\mathcal{W}(q)
$$

Then, recalling that $q_{0}=\mathcal{S}_{0}^{0^{-1}} \circ \mathcal{S}_{0}(q)$, we see that a necessary condition to satisfy $(11)$ is that $\left(q_{0} ; q\right)$ depends only on the first and higher derivatives of $q_{0}$. This in turn implies that for any constant $B$ we have $\left(q_{a}+B ; q_{b}\right)=\left(q_{a} ; q_{b}\right)$ that, together with (18), gives

$$
\left(q_{a}+B ; q_{b}\right)=\left(q_{a} ; q_{b}\right)=\left(q_{a} ; q_{b}+B\right) .
$$

Let $A$ be a non-vanishing constant and set $h(A, q)=(A q ; q)$. By (27) we have $h(A, q+B)=h(A, q)$, that is $h(A, q)$ is independent of $q$. On the other hand, by (25) $h(A, 0)=0$ that, together with (18), implies

$$
(A q ; q)=0=(q ; A q) .
$$

Eq. (20) implies $\left(q_{a} ; A q_{b}\right)=A^{-2}\left(\left(q_{a} ; q_{b}\right)-\left(A q_{b} ; q_{b}\right)\right)$, so that by $(28)$

$$
\left(q_{a} ; A q_{b}\right)=A^{-2}\left(q_{a} ; q_{b}\right) .
$$

By (18) and (29) we have

$$
\begin{gathered}
\left(A q_{a} ; q_{b}\right)=-A^{-2}\left(\partial_{q_{b}} q_{a}\right)^{2}\left(q_{b} ; A q_{a}\right) \\
=-\left(\partial_{q_{b}} q_{a}\right)^{2}\left(q_{b} ; q_{a}\right)=\left(q_{a} ; q_{b}\right),
\end{gathered}
$$

that is

$$
\left(A q_{a} ; q_{b}\right)=\left(q_{a} ; q_{b}\right) .
$$

Setting $f(q)=q^{-2}\left(q ; q^{-1}\right)$ and noticing that by (18) and (30) $f(A q)=-f\left(q^{-1}\right)$, we obtain

$$
\left(q ; q^{-1}\right)=0=\left(q^{-1} ; q\right) .
$$

Furthermore, since by (20) and (31) one has $\left(q_{a} ; q_{b}^{-1}\right)=$ $q_{b}^{4}\left(q_{a} ; q_{b}\right)$, it follows that

$$
\begin{aligned}
& \left(q_{a}^{-1} ; q_{b}\right)=-\left(\partial_{q_{b}} q_{a}^{-1}\right)^{2}\left(q_{b} ; q_{a}^{-1}\right) \\
& =-\left(\partial_{q_{b}} q_{a}\right)^{2}\left(q_{b} ; q_{a}\right)=\left(q_{a} ; q_{b}\right),
\end{aligned}
$$

so that

$$
\left(q_{a}^{-1} ; q_{b}\right)=\left(q_{a} ; q_{b}\right)=q_{b}^{-4}\left(q_{a} ; q_{b}^{-1}\right) .
$$

Since translations, dilatations and inversion are the generators of the Möbius group, it follows by (27)(29)(30) and (32) that

Lemma 1.Up to a coboundary term, Eq.(20) implies

$$
\begin{gathered}
\left(\gamma\left(q_{a}\right) ; q_{b}\right)=\left(q_{a} ; q_{b}\right), \\
\left(q_{a} ; \gamma\left(q_{b}\right)\right)=\left(\partial_{q_{b}} \gamma\left(q_{b}\right)\right)^{-2}\left(q_{a} ; q_{b}\right),
\end{gathered}
$$

where $\gamma(q)$ is an arbitrary $\operatorname{PSL}(2, \mathbb{C})$ transformation.

Now observe that since $\left(q_{a} ; q_{b}\right)$ should depend only on $\partial_{q_{b}}^{k} q_{a}, k \geq 1$, we have

$$
(q+\varepsilon f(q) ; q)=c_{1} \varepsilon f^{(k)}(q)+O\left(\varepsilon^{2}\right),
$$


where $q_{a}=q+\varepsilon f(q), q \equiv q_{b}$ and $f^{(k)} \equiv \partial_{q}^{k} f, k \geq 1$. Note that by lemma 1 and (33)

$$
\begin{gathered}
(A q+\varepsilon A f(q) ; A q)=(q+\varepsilon f(q) ; A q)= \\
=A^{-2}(q+\varepsilon f(q) ; q)=A^{-2} c_{1} \varepsilon f^{(k)}(q)+O\left(\varepsilon^{2}\right),
\end{gathered}
$$

on the other hand, setting $F(A q)=A f(q)$, by (33)

$$
\begin{gathered}
(A q+\varepsilon A f(q) ; A q)=(A q+\varepsilon F(A q) ; A q)= \\
=c_{1} \varepsilon \partial_{A q}^{k} F(A q)+O\left(\varepsilon^{2}\right)=A^{1-k} c_{1} \varepsilon f^{(k)}(q)+O\left(\varepsilon^{2}\right),
\end{gathered}
$$

that compared with (34) gives $k=3$. The above scaling property generalizes to higher order contributions in $\varepsilon$. In particular, at order $\varepsilon^{n}$ the quantity $(A q+\varepsilon A f(q) ; A q)$ is a sum of terms of the form

$$
\begin{aligned}
& c_{i_{1} \ldots i_{n}} \partial_{A q}^{i_{1}} \varepsilon F(A q) \cdots \partial_{A q}^{i_{n}} \varepsilon F(A q)= \\
= & c_{i_{1} \ldots i_{n}} \varepsilon^{n} A^{n-\sum i_{k}} f^{\left(i_{1}\right)}(q) \cdots f^{\left(i_{n}\right)}(q),
\end{aligned}
$$

and by (34) $\sum_{k=1}^{n} i_{k}=n+2$. On the other hand, since $\left(q_{a} ; q_{b}\right)$ depends only on $\partial_{q_{b}}^{k} q_{a}, k \geq 1$, we have

$$
i_{k} \geq 1, \quad k \in[1, n],
$$

so that either

$$
i_{k}=3, \quad i_{j}=1, \quad j \in[1, n], \quad j \neq k,
$$

or

$$
i_{k}=i_{j}=2, \quad i_{l}=1, \quad l \in[1, n], \quad l \neq k, l \neq j .
$$

Hence

$$
\begin{gathered}
(q+\varepsilon f(q) ; q)= \\
\sum_{n=1}^{\infty} \varepsilon^{n}\left(c_{n} f^{(3)} f^{(1)^{n-1}}+d_{n} f^{(2)^{2}} f^{(1)^{n-2}}\right), d_{1}=0 .
\end{gathered}
$$

Let us now consider the transformations

$$
q_{b}=v^{b a}\left(q_{a}\right), q_{c}=v^{c b}\left(q_{b}\right)=v^{c b} \circ v^{b a}\left(q_{a}\right), q_{c}=v^{c a}\left(q_{a}\right) .
$$

Note that $v^{a b}=v^{b a^{-1}}$, and

$$
v^{c a}=v^{c b} \circ v^{b a} .
$$

We can express these transformations in the form

$$
\begin{gathered}
q_{b}=q_{a}+\varepsilon^{b a}\left(q_{a}\right), \\
q_{c}=q_{b}+\varepsilon^{c b}\left(q_{b}\right)=q_{b}+\varepsilon^{c b}\left(q_{a}+\varepsilon^{b a}\left(q_{a}\right)\right), \\
q_{c}=q_{a}+\varepsilon^{c a}\left(q_{a}\right) .
\end{gathered}
$$

Since $q_{b}=q_{a}-\varepsilon^{a b}\left(q_{b}\right)$, we have $q_{b}=q_{a}-\varepsilon^{a b}\left(q_{a}+\varepsilon^{b a}\left(q_{a}\right)\right)$ that compared with $q_{b}=q_{a}+\varepsilon^{b a}\left(q_{a}\right)$ yields

$$
\varepsilon^{b a}+\varepsilon^{a b} \circ\left(\mathbf{1}+\varepsilon^{b a}\right)=0,
$$

where 1 denotes the identity map. More generally, Eq.(37) gives

$$
\varepsilon^{c a}\left(q_{a}\right)=\varepsilon^{c b}\left(q_{b}\right)+\varepsilon^{b a}\left(q_{a}\right)=\varepsilon^{c b}\left(q_{b}\right)-\varepsilon^{a b}\left(q_{b}\right),
$$

so that we obtain (36) with $v^{y x}=\mathbf{1}+\varepsilon^{y x}$

$$
\varepsilon^{c a}=\varepsilon^{c b} \circ\left(\mathbf{1}+\varepsilon^{b a}\right)+\varepsilon^{b a}=\left(\mathbf{1}+\varepsilon^{c b}\right) \circ\left(\mathbf{1}+\varepsilon^{b a}\right)-\mathbf{1} .
$$

Let us consider the case in which $\varepsilon^{y x}\left(q_{x}\right)=\varepsilon f_{y x}\left(q_{x}\right)$, with $\varepsilon$ infinitesimal. At first-order in $\varepsilon$ Eq.(38) reads

$$
\varepsilon^{c a}=\varepsilon^{c b}+\varepsilon^{b a}
$$

in particular, $\varepsilon^{a b}=-\varepsilon^{b a}$. Since $\left(q_{a} ; q_{b}\right)=c_{1} \varepsilon^{a b^{\prime \prime \prime}}\left(q_{b}\right)+$ $O^{a b}\left(\varepsilon^{2}\right)$, where ' denotes the derivative with respect to the argument, we can use the cocycle condition (20) to get

$$
c_{1} \varepsilon^{a c \prime \prime \prime}\left(q_{c}\right)+O^{a c}\left(\varepsilon^{2}\right)=\left(1+\varepsilon^{b c^{\prime}}\left(q_{c}\right)\right)^{2}
$$

$$
\times\left(c_{1} \varepsilon^{a b^{\prime \prime \prime}}\left(q_{b}\right)+O^{a b}\left(\varepsilon^{2}\right)-c_{1} \varepsilon^{c b^{\prime \prime \prime}}\left(q_{b}\right)-O^{c b}\left(\varepsilon^{2}\right)\right),
$$

that at first-order in $\varepsilon$ corresponds to (39). We see that $c_{1} \neq 0$. For, if $c_{1}=0$, then by (40), at second-order in $\varepsilon$ one would have

$$
O^{a c}\left(\varepsilon^{2}\right)=O^{a b}\left(\varepsilon^{2}\right)-O^{c b}\left(\varepsilon^{2}\right),
$$

which contradicts (39). In fact, by (35) we have

$$
O^{a b}\left(\varepsilon^{2}\right)=c_{2} \varepsilon^{a b^{\prime \prime \prime}}\left(q_{b}\right) \varepsilon^{a b^{\prime}}\left(q_{b}\right)+d_{2} \varepsilon^{a b^{\prime \prime 2}}\left(q_{b}\right)+O^{a b}\left(\varepsilon^{3}\right),
$$

that together with (41) provides a relation which cannot be consistent with $\varepsilon^{a c}\left(q_{c}\right)=\varepsilon^{a b}\left(q_{b}\right)-\varepsilon^{c b}\left(q_{b}\right)$. A possibility is that $\left(q_{a} ; q_{b}\right)=0$. However, this is ruled out by the EP, so that

$$
c_{1} \neq 0 \text {. }
$$

Higher-order contributions due to a non-vanishing $c_{1}$ are obtained by using

$$
q_{c}=q_{b}+\varepsilon^{c b}\left(q_{b}\right), \quad \varepsilon^{a c}\left(q_{c}\right)=\varepsilon^{a b}\left(q_{b}\right)-\varepsilon^{c b}\left(q_{b}\right),
$$

and $\varepsilon^{b c}\left(q_{c}\right)=-\varepsilon^{c b}\left(q_{b}\right)$ in $c_{1} \partial_{q_{c}}^{3} \varepsilon^{a c}\left(q_{c}\right)$ and in

$$
c_{1}\left(2 \partial_{q_{c}} \varepsilon^{b c}\left(q_{c}\right)+\partial_{q_{c}} \varepsilon^{b c}\left(q_{c}\right)^{2}\right) \partial_{q_{b}}^{3}\left(\varepsilon^{a b}\left(q_{b}\right)-\varepsilon^{c b}\left(q_{b}\right)\right) .
$$

Note that one can also consider the case in which both the first- and second-order contributions to $\left(q_{a} ; q_{b}\right)$ are vanishing. However, this possibility is ruled out by a similar analysis. In general, one has that if the first non-vanishing contribution to $\left(q_{a} ; q_{b}\right)$ is of order $\varepsilon^{n}, n \geq 2$, then, unless $\left(q_{a} ; q_{b}\right)=0$, the cocycle condition (20) cannot be consistent with the linearity of (39). Observe that we proved that $c_{1} \neq 0$ is a necessary condition for the existence of solutions $\left(q_{a} ; q_{b}\right)$ of the cocycle 
condition (20), depending only on the first and higher derivatives of $q_{a}$. Existence of solutions follows from the fact that the Schwarzian derivative $\left\{q_{a}, q_{b}\right\}$ solves (20) and depends only on the first and higher derivatives of $q_{a}$.

The fact that $c_{1}=0$ implies $\left(q_{a} ; q_{b}\right)=0$, can be also seen by explicitly evaluating the coefficients $c_{n}$ and $d_{n}$. These can be obtained using the same procedure considered above to prove that $c_{1} \neq 0$. Namely, inserting the expansion (35) in (20) and using $q_{c}=q_{b}+\varepsilon^{c b}\left(q_{b}\right), \varepsilon^{a c}\left(q_{c}\right)=\varepsilon^{a b}\left(q_{b}\right)-\varepsilon^{c b}\left(q_{b}\right)$ and $\varepsilon^{b c}\left(q_{c}\right)=-\varepsilon^{c b}\left(q_{b}\right)$, we obtain

$$
c_{n}=(-1)^{n-1} c_{1}, \quad d_{n}=\frac{3}{2}(-1)^{n-1}(n-1) c_{1},
$$

which in fact are the coefficients one obtains expanding $c_{1}\{q+\varepsilon f(q), q\}$. However, we now use only the fact that $c_{1} \neq 0$, as the relation $(q+\varepsilon f(q) ; q)=c_{1}\{q+\varepsilon f(q), q\}$ can be proved without making the calculations leading to (42). Summarizing, we have

\section{Lemma 2.If}

$$
q_{a}=q_{b}+\varepsilon^{a b}\left(q_{b}\right),
$$

the unique solution of Eq.(20), depending only on the first and higher derivatives of $q_{a}$, is

$$
\left(q_{a} ; q_{b}\right)=c_{1} \varepsilon^{a b^{\prime \prime \prime}}\left(q_{b}\right)+O^{a b}\left(\varepsilon^{2}\right), \quad c_{1} \neq 0 .
$$

It is now easy to prove that, up to a multiplicative constant and a coboundary term, the Schwarzian derivative is the unique solution of the cocycle condition (20). Let us first note that

$$
\left[q_{a} ; q_{b}\right]=\left(q_{a} ; q_{b}\right)-c_{1}\left\{q_{a} ; q_{b}\right\}
$$

satisfies the cocycle condition

$$
\left[q_{a} ; q_{c}\right]=\left(\partial_{q_{c}} q_{b}\right)^{2}\left(\left[q_{a} ; q_{b}\right]-\left[q_{c} ; q_{b}\right]\right) .
$$

In particular, since both $\left(q_{a} ; q_{b}\right)$ and $\left\{q_{a} ; q_{b}\right\}$ depend only on the first and higher derivatives of $q_{a}$, we have, as in the case of $(q+\varepsilon f(q) ; q)$, that

$$
[q+\varepsilon f(q) ; q]=\tilde{c}_{1} \varepsilon f^{(3)}(q)+O\left(\varepsilon^{2}\right),
$$

where either $\tilde{c}_{1} \neq 0$ or $[q+\varepsilon f(q) ; q]=0$. However, since $\{q+$ $\varepsilon f(q) ; q\}=\varepsilon f^{(3)}(q)+O\left(\varepsilon^{2}\right)$ and $(q+\varepsilon f(q) ; q)=\varepsilon f^{(3)}(q)+$ $O\left(\varepsilon^{2}\right)$, we have $\tilde{c}_{1}=0$ and the Lemma yields $[q+\varepsilon f(q) ; q]=$ 0 . Therefore, we have that the EP univocally implies that

$$
\left(q_{a} ; q_{b}\right)=-\frac{\beta^{2}}{4 m}\left\{q_{a}, q_{b}\right\}
$$

where for convenience we replaced $c_{1}$ by $-\beta^{2} / 4 m$. This concludes the proof of theorem 1 .

We observe that despite some claims [5][6], we have not be able to find in the literature a complete and close proof of the above theorem. We thank D.B. Fuchs for a bibliographic comment concerning the above theorem.
In deriving the equivalence of states we considered the case of one-particle states with identical masses. The generalization to the case with different masses is straightforward. In particular, the right hand side of Eq.(20) gets multiplied by $m_{b} / m_{a}$, so that the cocycle condition becomes

$$
m_{a}\left(q_{a} ; q_{c}\right)=m_{a}\left(\partial_{q_{c}} q_{b}\right)^{2}\left(q_{a} ; q_{b}\right)+m_{b}\left(q_{b} ; q_{c}\right)
$$

explicitly showing that the mass appears in the denominator and that it refers to the label in the first entry of $(\cdot ; \cdot)$, that is

$$
\left(q_{a} ; q_{b}\right)=-\frac{\hbar^{2}}{4 m_{a}}\left\{q_{a} ; q_{b}\right\}
$$

The QSHJE (21) follows almost immediately by (43) [1].

The above investigation may be applied to CFT. Let us consider a local conformal transformation of the stress tensor in a 2D CFT. The infinitesimal variation of $T$ is given by

$$
\delta_{\varepsilon} T(w)=-\frac{1}{12} c \partial_{w}^{3} \varepsilon(w)-2 T(w) \partial_{w} \varepsilon(w)-\varepsilon(w) \partial_{w} T(w),
$$

where $c$ is the central charge. The finite version of such a transformation is

$$
\tilde{T}(w)=\left(\partial_{w} z\right)^{2} T(z)+\frac{c}{12}\{w, z\}
$$

While it is immediate to see that (45) implies (44), the viceversa is not evident. A possible way to prove (45) is just to set

$$
\tilde{T}(w)=\left(\partial_{w} z\right)^{2} T(z)+k(w ; z)
$$

and then to impose the cocycle condition which will show that $(w ; z)$ is proportional to $\{w, z\}$. Comparison with the infinitesimal transformation (44) fixes the constant $k$.

In [2] it has been shown that the cocycle condition fixes the higher dimensional version of the Schwarzian derivative. In this respect we observe that its definition seems an open question in mathematical literature. While in the one dimensional case the QSHJE reduces to a unique differential equation, this is not immediate in the higher dimensional case. However, it turns out that such a reduction exists upon introducing an antisymmetric tensor [2] (in this respect it is worth noticing that some author introduces a connection to define the higher dimensional Schwarzian derivative).

A basic feature of the cocycle condition is that it implies, as it should, the higher dimensional Möbious invariance with respect to $q_{a}$ in $\left(q_{a} ; q_{b}\right)$ (with similar properties with respect to $q_{b}$ ). In particular, in [2] it has been shown that

$$
\left(q^{a} ; q^{b}\right)=-\frac{\hbar^{2}}{2 m}\left[\left(p^{b} \mid p^{a}\right) \frac{\Delta^{a} R^{a}}{R^{a}}-\frac{\Delta^{b} R^{b}}{R^{b}}\right] .
$$

It would be interesting to consider such a definition in the context of the transformation properties of the stress tensor in higher dimensional CFTs. 


\section{PROOF OF THEOREM 2}

The QSHJE is equivalent to

$$
\{w, q\}=-\frac{4 m}{\hbar^{2}} \mathcal{W}(q),
$$

where $w=\psi^{D} / \psi$ with $\psi^{D}$ and $\psi$ two real linearly independent solutions of the Schrödinger equation. Existence of this equation requires some conditions on the continuity properties of $w$ and its derivatives. Since the QSHJE is the consequence of the EP, we can say that the EP imposes some constraints on $w=\psi^{D} / \psi$. These constraints are nothing but the existence of the QSHJE (21) or, equivalently, of Eq.(48). That is, implementation of the EP imposes that $\{w, q\}$ exists, so that

$$
w \neq \text { cnst }, w \in C^{2}(\mathbb{R}), \text { and } \partial_{q}^{2} w \text { different. on } \mathbb{R} .
$$

These conditions are not complete. The reason is that, as we have seen, the implementation of the EP requires that the properties of the Schwarzian derivative be satisfied. Actually, its very properties, derived from the EP, led to the identification $\left(q_{a} ; q_{b}\right)=-\hbar^{2}\left\{q_{a}, q_{b}\right\} / 4 m$. Therefore, in order to implement the EP the transformation properties of the Schwarzian derivative and its symmetries must be satisfied. In deriving the transformation properties of $\left(q_{a} ; q_{b}\right)$ we noticed how, besides dilatations and translations, there is a highly non-trivial symmetry such as that under inversion. Therefore, we have that (48) must be equivalent to

$$
\left\{w^{-1}, q\right\}=-\frac{4 m}{\hbar^{2}} \mathcal{W}(q) .
$$

A property of the Schwarzian derivative is duality between its entries

$$
\{w, q\}=-\left(\frac{\partial w}{\partial q}\right)^{2}\{q, w\} .
$$

This shows that the invariance under inversion of $w$ reflects in the invariance, up to a Jacobian factor, under inversion of $q$. That is $\left\{w, q^{-1}\right\}=q^{4}\{w, q\}$, so that the QSHJE (48) can be written in the equivalent form

$$
\left\{w, q^{-1}\right\}=-\frac{4 m}{\hbar^{2}} q^{4} \mathcal{W}(q) .
$$

In other words, starting from the EP one can arrive to either Eq.(48) or Eq.(51). The consequence of this fact is that since under

$$
q \rightarrow \frac{1}{q}
$$

$0^{ \pm}$maps to $\pm \infty$, we have to extend (49) to the point at infinity. In other words, (49) should hold on the extended real line $\hat{\mathbb{R}}=\mathbb{R} \cup\{\infty\}$. This aspect is related to the fact that the Möbius transformations, under which the Schwarzian derivative transforms as a quadratic differential, map circles to circles. We stress that we are considering the systems defined on $\mathbb{R}$ and not $\hat{\mathbb{R}}$. What happens is that the existence of QSHJE forces us to impose smoothly joining conditions even at $\pm \infty$, that is (49) must be extended to

$$
w \neq \text { cnst }, w \in C^{2}(\hat{\mathbb{R}}), \text { and } \partial_{q}^{2} w \text { different. on } \hat{\mathbb{R}} .
$$

One may easily check that $w$ is a Möbius transformation of the trivializing map [1]. Therefore, Eq.(50), which is defined if and only if $w(q)$ can be inverted, that is if $\partial_{q} w \neq 0, \forall q \in \mathbb{R}$, is a consequence of the cocycle condition (19). By (51) we see that also local univalence should be extended to $\hat{\mathbb{R}}$. This implies the following joining condition at spatial infinity

$$
w(-\infty)= \begin{cases}w(+\infty), & \text { for } w(-\infty) \neq \pm \infty \\ -w(+\infty), & \text { for } w(-\infty)= \pm \infty\end{cases}
$$

As illustrated by the non-univalent function $w=q^{2}$, the apparently natural choice $w(-\infty)=w(+\infty)$, one would consider also in the $w(-\infty)= \pm \infty$ case, does not satisfy local univalence.

We saw that the EP implied the QSHJE (21). However, although this equation implies the SE, we saw that there are aspects concerning the canonical variables which arise in considering the QSHJE rather than the SE. In this respect a natural question is whether the basic facts of QM also arise in our formulation. A basic point concerns a property of many physical systems such as energy quantization. This is a matter of fact beyond any interpretational aspect of QM. Then, as we used the EP to get the QSHJE, it is important to understand how energy quantization arises in our approach. According to the EP, the QSHJE contains all the possible information on a given system. Then, the QSHJE itself should be sufficient to recover the energy quantization including its structure. In the usual approach the quantization of the spectrum arises from the basic condition that in the case in which $\lim _{q \rightarrow \pm \infty} \mathcal{W}>0$, the wave-function should vanish at infinity. Once the possible solutions are selected, one also imposes the continuity conditions whose role in determining the possible spectrum is particularly transparent in the case of discontinuous potentials. For example, in the case of the potential well, besides the restriction on the spectrum due to the $L^{2}(\mathbb{R})$ condition for the wave-function (a consequence of the probabilistic interpretation of the wave-function), the spectrum is further restricted by the smoothly joining conditions. Since the SE contains the term $\partial_{q}^{2} \psi$, the continuity conditions correspond to an existence condition for this equation. On the other hand, also in this case, the physical reason underlying this request is the interpretation of the wave-function in terms of probability amplitude. Actually, strictly speaking, the continuity conditions come from the continuity of the probability density $\rho=|\psi|^{2}$. This density should also satisfy the continuity equation $\partial_{t} \rho+\partial_{q} j=0$, where $j=i \hbar\left(\psi \partial_{q} \bar{\psi}-\bar{\psi} \partial_{q} \psi\right) / 2 m$. Since for stationary states $\partial_{t} \rho=0$, it follows that in this case $j=c n s t$. Therefore, in the usual formulation, it is just the interpretation of the wave-function in terms of probability amplitude, with the consequent meaning of $\rho$ and $j$, which provides the physical motivation for imposing the continuity of the wavefunction and of its first derivative.

Now observe that in our formulation the continuity conditions arise from the QSHJE. In fact, (52) implies continuity of 
$\psi^{D}, \psi$, with $\partial_{q} \psi^{D}$ and $\partial_{q} \psi$ differentiable, that is

$$
E P \rightarrow\left(\psi^{D}, \psi\right) \text { cont } . \text { and }\left(\psi^{D^{\prime}}, \psi^{\prime}\right) \text { different. . }
$$

In the following we will see that if $V(q)>E, \forall q \in \mathbb{R}$, then there are no solutions such that the ratio of two real linearly independent solutions of the SE corresponds to a local selfhomeomorphism of $\hat{\mathbb{R}}$. The fact that this is an unphysical situation can be also seen from the fact that the case $V>E$, $\forall q \in \mathbb{R}$, has no classical limit. Therefore, if $V>E$ both at $-\infty$ and $+\infty$, a physical situation requires that there are at least two points where $V-E=0$. More generally, if the potential is not continuous, $V(q)-E$ should have at least two turning points. Let us denote by $q_{-}\left(q_{+}\right)$the lowest (highest) turning point. Note that by (23) we have

$$
\int_{q_{-}}^{-\infty} d x \kappa(x)=-\infty, \quad \int_{q_{+}}^{+\infty} d x \kappa(x)=+\infty
$$

where $\kappa=\sqrt{2 m(V-E)} / \hbar$. Before going further, let us stress that what we actually need to prove is that, in the case (23), the joining condition (53) requires that the corresponding SE has an $L^{2}(\mathbb{R})$ solution. Observe that while (52), which however follows from the EP, can be recognized as the standard condition (54), the other condition (53), which still follows from the existence of the QSHJE, and therefore from the EP, is not directly recognized in the standard formulation. Since this leads to energy quantization, while in the usual approach one needs one more assumption, we see that there is a quite fundamental difference between the QSHJE and the SE. We stress that (52) and (53) guarantee that $w$ is a local self-homeomorphism of $\hat{\mathbb{R}}$.

Let us first show that the request that the corresponding SE has an $L^{2}(\mathbb{R})$ solution is a sufficient condition for $w$ to satisfy (53). Let $\psi \in L^{2}(\mathbb{R})$ and denote by $\psi^{D}$ a linearly independent solution. As we will see, the fact that $\psi^{D} \propto \psi \psi$ implies that if $\psi \in L^{2}(\mathbb{R})$, then $\psi^{D} \notin L^{2}(\mathbb{R})$. In particular, $\psi^{D}$ is divergent both at $q=-\infty$ and $q=+\infty$. Let us consider the real ratio

$$
w=\frac{A \psi^{D}+B \psi}{C \psi^{D}+D \psi},
$$

where $A D-B C \neq 0$. Since $\psi \in L^{2}(\mathbb{R})$, we have

$$
\lim _{q \rightarrow \pm \infty} w=\lim _{q \rightarrow \pm \infty} \frac{A \psi^{D}+B \psi}{C \psi^{D}+D \psi}=\frac{A}{C},
$$

that is $w(-\infty)=w(+\infty)$. In the case in which $C=0$ we have

$$
\lim _{q \rightarrow \pm \infty} w=\lim _{q \rightarrow \pm \infty} \frac{A \psi^{D}}{D \psi}= \pm \varepsilon \cdot \infty,
$$

where $\varepsilon= \pm 1$. The fact that $\lim _{q \rightarrow \pm \infty} A \psi^{D} / D \psi$ diverges follows from the mentioned properties of $\psi^{D}$ and $\psi$. It remains to check that if $\lim _{q \rightarrow-\infty} A \psi^{D} / D \psi=-\infty$, then $\lim _{q \rightarrow+\infty} A \psi^{D} / D \psi=+\infty$, and vice versa. This can be seen by observing that

$$
\psi^{D}(q)=c \psi(q) \int_{q_{0}}^{q} d x \psi^{-2}(x)+d \psi(q)
$$

$c \in \mathbb{R} \backslash\{0\}, d \in \mathbb{R}$. Since $\psi \in L^{2}(\mathbb{R})$ we have $\psi^{-1} \notin$ $L^{2}(\mathbb{R})$ and $\int_{q_{0}}^{+\infty} d x \psi^{-2}(x)=+\infty, \int_{q_{0}}^{-\infty} d x \psi^{-2}(x)=-\infty$, so that $\psi^{D}(-\infty) / \psi(-\infty)=-\varepsilon \cdot \infty=-\psi^{D}(+\infty) / \psi(+\infty)$, where $\varepsilon=\operatorname{sgn} c$.

We now show that the existence of an $L^{2}(\mathbb{R})$ solution of the SE is a necessary condition to satisfy the joining condition (53). We give two different proofs of this, one is based on the WKB approximation while the other one uses Wronskian arguments. In the WKB approximation, we have

$$
\psi=\frac{A_{-}}{\sqrt{\kappa}} e^{-\int_{q_{-}}^{q} d x \kappa(x)}+\frac{B_{-}}{\sqrt{\kappa}} e^{\int_{q_{-}}^{q} d x \kappa(x)}, \quad q \ll q_{-},
$$

and

$$
\psi=\frac{A_{+}}{\sqrt{\kappa}} e^{-\int_{q+}^{q} d x \kappa(x)}+\frac{B_{+}}{\sqrt{\kappa}} e^{\int_{q+}^{q} d x \kappa(x)}, \quad q \gg q_{+} .
$$

In the same approximation, a linearly independent solution has the form

$$
\psi^{D}=\frac{A_{-}^{D}}{\sqrt{\kappa}} e^{-\int_{q_{-}}^{q} d x \kappa(x)}+\frac{B_{-}^{D}}{\kappa} e^{\int_{q_{-}}^{q} d x \kappa(x)}, \quad q \ll q_{-} .
$$

Similarly, in the $q \gg q_{+}$region we have

$$
\psi^{D}=\frac{A_{+}^{D}}{\sqrt{\kappa}} e^{-\int_{q_{+}}^{q} d x \kappa(x)}+\frac{B_{+}^{D}}{\sqrt{\kappa}} e^{\int_{q_{+}}^{q} d x \kappa(x)}, \quad q \gg q_{+} .
$$

Note that (56) and (57) are derived by solving the differential equations corresponding to the WKB approximation for $q \ll q_{-}$and $q \gg q_{+}$, so that the coefficients of $\kappa^{-1 / 2} \exp \pm \int_{q_{-}}^{q} d x \kappa(x)$, e.g. $A_{-}$and $B_{-}$in (56), cannot be simultaneously vanishing. In particular, the fact that $\psi^{D} \propto \psi$ yields

$$
A_{-} B_{-}^{D}-A_{-}^{D} B_{-} \neq 0, \quad A_{+} B_{+}^{D}-A_{+}^{D} B_{+} \neq 0 .
$$

Let us now consider the case in which, for a given $E$ satisfying (23), any solution of the corresponding SE diverges at least at one of the two spatial infinities, that is

$$
\lim _{q \rightarrow+\infty}(|\psi(-q)|+|\psi(q)|)=+\infty .
$$

This implies that there is a solution diverging both at $q=$ $-\infty$ and $q=+\infty$. In fact, if two solutions $\psi_{1}$ and $\psi_{2}$ satisfy $\psi_{1}(-\infty)= \pm \infty, \psi_{1}(+\infty) \neq \pm \infty$ and $\psi_{2}(-\infty) \neq \pm \infty$, $\psi_{2}(+\infty)= \pm \infty$, then $\psi_{1}+\psi_{2}$ diverges at $\pm \infty$. On the other hand, (58) rules out the case in which all the solutions in their WKB approximation are divergent only at one of the two spatial infinities, say $-\infty$. Since, in the case (23), a solution which diverges in the WKB approximation is itself divergent (and vice versa), we have that in the case (23), the fact that all the solutions of the SE diverge only at one of the two spatial infinities cannot occur.

Let us denote by $\psi$ a solution which is divergent both at $-\infty$ and $+\infty$. In the WKB approximation this means that both $A_{-}$ and $B_{+}$are non-vanishing, so that

$$
\psi_{q \rightarrow-\infty}^{\sim} \frac{A_{-}}{\sqrt{\kappa}} e^{-\int_{q_{-}}^{q} d x \kappa}, \quad \psi_{q \rightarrow+\infty}^{\sim} \frac{B_{+}}{\sqrt{\kappa}} e^{\int_{q_{+}}^{q} d x \kappa} .
$$


The asymptotic behavior of the ratio $\psi^{D} / \psi$ is given by

$$
\lim _{q \rightarrow-\infty} \frac{\psi^{D}}{\psi}=\frac{A_{-}^{D}}{A_{-}}, \quad \lim _{q \rightarrow+\infty} \frac{\psi^{D}}{\psi}=\frac{B_{+}^{D}}{B_{+}} .
$$

Note that since in the case at hand any divergent solution also diverges in the WKB approximation, we have that (59) rules out the case $A_{-}^{D}=B_{+}^{D}=0$. Let us then suppose that either $A_{-}^{D}=0$ or $B_{+}^{D}=0$. If $A_{-}^{D}=0$, then $w(-\infty)=0 \neq w(+\infty)$. Similarly, if $B_{+}^{D}=0$, then $w(+\infty)=0 \neq w(-\infty)$. Hence, in this case $w$, and therefore the trivializing map, cannot satisfy (53). On the other hand, also in the case in which both $A_{-}^{D}$ and $B_{+}^{D}$ are non-vanishing, $w$ cannot satisfy Eq.(53). For, if $A_{-}^{D} / A_{-}=B_{+}^{D} / B_{+}$, then

$$
\phi=\psi-\frac{A_{-}}{A_{-}^{D}} \psi^{D}=\psi-\frac{B_{+}}{B_{+}^{D}} \psi^{D},
$$

would be a solution of the SE whose WKB approximation has the form

$$
\phi=\frac{B_{-}}{\sqrt{\kappa}} e^{\int_{q_{-}}^{q} d x \kappa(x)}, \quad q \ll q_{-}
$$

and

$$
\phi=\frac{A_{+}}{\sqrt{\kappa}} e^{-\int_{q_{+}}^{q} d x \kappa(x)}, \quad q \gg q_{+} .
$$

Hence, if $A_{-}^{D} / A_{-}=B_{+}^{D} / B_{+}$, then there is a solution whose WKB approximation vanishes both at $-\infty$ and $+\infty$. On the other hand, we are considering the values of $E$ satisfying Eq.(23) and for which any solution of the SE has the property (59). This implies that no solutions can vanish both at $-\infty$ and $+\infty$ in the WKB approximation. Hence

$$
\frac{A_{-}^{D}}{A_{-}} \neq \frac{B_{+}^{D}}{B_{+}},
$$

so that $w(-\infty) \neq w(+\infty)$. We also note that not even the case $w(-\infty)= \pm \infty=-w(+\infty)$ can occur, as this would imply that $A_{-}=B_{+}=0$, which in turn would imply, against the hypothesis, that there are solutions vanishing at $q= \pm \infty$. Hence, if for a given $E$ satisfying (23), any solution of the corresponding SE diverges at least at one of the two spatial infinities, we have that the trivializing map has a discontinuity at $q= \pm \infty$. As a consequence, the EP cannot be implemented in this case so that this value $E$ cannot belong to the physical spectrum.

Therefore, the physical values of $E$ satisfying (23) are those for which there are solutions which are divergent neither at $-\infty$ nor at $+\infty$. On the other hand, from the WKB approximation and (23), it follows that the non-divergent solutions must vanish both at $-\infty$ and $+\infty$. It follows that the only energy levels satisfying the property (23), which are compatible with the EP, are those for which there exists the solution vanishing both at $\pm \infty$. On the other hand, solutions vanishing as $\kappa^{-1 / 2} \exp \int_{q_{-}}^{q} d x \kappa$ at $-\infty$ and $\kappa^{-1 / 2} \exp -\int_{q_{+}}^{q} d x \kappa$ at $+\infty$, with $P_{ \pm}^{2}>0$, cannot contribute with an infinite value to $\int_{-\infty}^{+\infty} d x \psi^{2}(x)$. The reason is that existence of the QSHJE requires that $\left\{e^{\frac{2 i}{\hbar} S_{0}}, q\right\}$ be defined and this, in turn, implies that any solution of the SE must be continuous. On the other hand, since $\psi$ is continuous, and therefore finite also at finite values of $q$, we have $\int_{q_{a}}^{q_{b}} d x \psi^{2}(x)<+\infty$ for all finite $q_{a}$ and $q_{b}$. In other words, the only possibility for a continuous function to have a divergent value of $\int_{-\infty}^{+\infty} d x \psi^{2}(x)$ comes from its behavior at $\pm \infty$. Therefore, since the implementation of the EP in the case (23) requires that the corresponding $E$ should admit a solution with the behavior

$$
\psi_{q \rightarrow-\infty}^{\sim} \frac{A_{-}}{\sqrt{\kappa}} e^{\int_{q_{-}}^{q} d x \kappa}, \quad \psi_{q \rightarrow+\infty}^{\sim} \frac{B_{+}}{\sqrt{\kappa}} e^{-\int_{q_{+}}^{q} d x \kappa},
$$

we have the following basic fact

The values of E satisfying

$$
V(q)-E \geq \begin{cases}P_{-}^{2}>0, & q<q_{-}, \\ P_{+}^{2}>0, & q>q_{+},\end{cases}
$$

are physically admissible if and only if the corresponding $S E$ has an $L^{2}(\mathbb{R})$ solution.

We now give another proof of the fact that if $\mathcal{W}$ is of the type (60), then the corresponding SE must have an $L^{2}(\mathbb{R})$ solution in order to satisfy (53). In particular, we will show that this is a necessary condition. That this is sufficient has been already proved above.

By Wronskian arguments, which can be found in Messiah's book [7], imply that if $V(q)-E \geq P_{+}^{2}>0, q>q_{+}$, then as $q \rightarrow+\infty$, we have $\left(P_{+}>0\right)$

- There is a solution of the SE that vanishes at least as $e^{-P_{+} q}$.

- Any other linearly independent solution diverges at least as $e^{P+q}$.

Similarly, if $V(q)-E \geq P_{-}^{2}>0, q<q_{-}$, then as $q \rightarrow-\infty$, we have $\left(P_{-}>0\right)$

- There is a solution of the SE that vanishes at least as $e^{P_{-} q}$.

- Any other linearly independent solution diverges at least as $e^{-P_{-} q}$.

These properties imply that if there is a solution of the SE in $L^{2}(\mathbb{R})$, then any solution is either in $L^{2}(\mathbb{R})$ or diverges both at $-\infty$ and $+\infty$. Let us show that the possibility that a solution vanishes only at one of the two spatial infinities is ruled out. Suppose that, besides the $L^{2}(\mathbb{R})$ solution, which we denote by $\psi_{1}$, there is a solution $\psi_{2}$ which is divergent only at $+\infty$. On the other hand, the above properties show that there exists also a solution $\psi_{3}$ which is divergent at $-\infty$. Since the number of linearly independent solutions of the SE is two, we have $\psi_{3}=A \psi_{1}+B \psi_{2}$. However, since $\psi_{1}$ vanishes both at $-\infty$ and $+\infty$, we see that $\psi_{3}=A \psi_{1}+B \psi_{2}$ can be satisfied only if $\psi_{2}$ and $\psi_{3}$ are divergent both at $-\infty$ and $+\infty$. This fact and the above properties imply that

If the SE has an $L^{2}(\mathbb{R})$ solution, then any solution has two possible asymptotics 
- Vanishes both at $-\infty$ and $+\infty$ at least as $e^{P_{-} q}$ and $e^{-P_{+} q}$ respectively.

- Diverges both at $-\infty$ and $+\infty$ at least as $e^{-P_{-} q}$ and $e^{P_{+} q}$ respectively.

Similarly, we have

If the $S E$ does not admit an $L^{2}(\mathbb{R})$ solution, then any solution has three possible asymptotics

- Diverges both at $-\infty$ and $+\infty$ at least as $e^{-P_{-} q}$ and $e^{P_{+} q}$ respectively.

- Diverges at $-\infty$ at least as $e^{-P_{-} q}$ and vanishes at $+\infty$ at least as $e^{-P_{+} q}$

- Vanishes at $-\infty$ at least as $e^{P_{-} q}$ and diverges at $+\infty$ at least as $e^{P_{+} q}$.

Let us consider the ratio $w=\psi^{D} / \psi$ in the latter case. Since any different choice of linearly independent solutions of the SE corresponds to a Möbius transformation of $w$, we can choose

$$
\psi_{q \rightarrow-\infty}^{D} a_{-} e^{P_{-} q}, \quad \psi_{q \rightarrow+\infty}^{D} \underset{\sim}{\sim} a_{+} e^{P_{+} q},
$$

and

$$
\psi_{q \rightarrow-\infty}^{\sim} b_{-} e^{-P_{-} q}, \quad \psi_{q \rightarrow+\infty}^{\sim} b_{+} e^{-P_{+} q},
$$

were by $\sim$ we mean that $\psi^{D}$ and $\psi$ either diverge or vanish "at least as". Their ratio has the asymptotic

$$
\frac{\psi^{D}}{\psi} \underset{q \rightarrow-\infty}{\sim} c_{-} e^{2 P_{-} q} \rightarrow 0, \quad \frac{\psi^{D}}{\psi} \underset{q \rightarrow+\infty}{\sim} c_{+} e^{2 P_{+} q} \rightarrow \pm \infty,
$$

so that $w$ cannot satisfy Eq.(53). This concludes the alternative proof of the fact that, in the case (60), the existence of the $L^{2}(\mathbb{R})$ solution is a necessary condition in order (53) be satisfied. The fact that this is a sufficient condition has been proved previously in deriving Eq.(55).

The above results imply that the usual quantized spectrum arises as a consequence of the EP.

Let us note that we are considering real solutions of the SE. Thus, apparently, in requiring the existence of an $L^{2}(\mathbb{R})$ solution, one should specify the existence of a real $L^{2}(\mathbb{R})$ solution. However, if there is an $L^{2}(\mathbb{R})$ solution $\psi$, this is unique up to a constant, and since also $\bar{\psi} \in L^{2}(\mathbb{R})$ solves the SE, we have that an $L^{2}(\mathbb{R})$ solution of the SE is real up to a phase.

\section{THE TWO-PARTICLE MODEL}

The EP leads to the introduction of length scales [1][2][3], a fact related to the nontriviality of the quantum potential, even in the case of $\mathcal{W}^{0}$. We note that also $\mathcal{S}_{0}$, as follows by the EP, is never trivial, in particular

$$
\mathcal{S}_{0} \neq \text { cnst }, \quad \forall \mathcal{W} \in \mathcal{H} .
$$

The QSHJE (21), first investigated by Floyd in a series of important papers [8], has been studied and reviewed by several authors [9][10].

The real solution of the QSHJE (21) is

$$
e^{\frac{2 i}{\hbar} S_{0}\{\delta\}}=e^{i \alpha} \frac{w+i \bar{\ell}}{w-i \ell}, \quad \delta \equiv\{\alpha, \ell\},
$$

with $\alpha \in \mathbb{R}$ and $\ell=\ell_{1}+i \ell_{2}, \ell_{1} \neq 0$, are integration constants. The condition $\ell_{1} \neq 0$ is necessary for $\mathcal{S}_{0}$ and the quantum potential $Q$ to be well-defined.

The formulation has a manifest duality between real pairs of linearly independent solutions [1], a property strictly related to Legendre duality [11] (see [12] for related issues). A similar structure also appears in uniformization theory of Riemann surfaces [1][4]. Whereas in the standard approach one usually considers only one solution of the SE, i.e. the wave-function itself, in our formulation the relevant formulas contain the linear combination $\psi^{D}+i \ell \psi$. Since $\ell_{1} \neq 0, \psi^{D}$ and $\psi$ appears always in pair. So, Legendre duality, nontriviality of $\mathcal{S}_{0}$ and $Q$ are deeply related features which are direct consequences of the EP. In turn, these properties imply the appearance of fundamental constants such as the Planck length [1][2][3]. The simplest way to see this is to consider the SE in the trivial case, that is $\partial_{q_{0}}^{2} \psi=0$, so that $\psi^{D}=q_{0}, \psi=1$ and the typical combination reads $q_{0}+i \ell_{0}$, implying that $\ell_{0} \equiv \ell$ should have the dimension of a length. The fact that $\ell$ has the dimension of a length is true for any state. Since $\ell_{0}$ appears in the QSHJE with $\mathcal{W}^{0} \equiv 0$, the system does not provide any dimensional quantity, so that we have to introduce some fundamental length. The appearance of fundamental constants also arises in considering the limits $\hbar \rightarrow 0$ and $E \rightarrow 0$ in the case of a free particle [1]. So, for example, a consistent expression for the quantum potential associated to the trivial state $\mathcal{W}^{0}$, which vanishes as $\hbar \rightarrow 0$, is

$$
Q^{0}=\frac{\hbar}{4 m}\left\{S_{0}^{0}, q_{0}\right\}=-\frac{\hbar^{3} G}{2 m c^{3}} \frac{1}{\left|q_{0}-i \lambda_{p}\right|^{4}},
$$

where

$$
\lambda_{p}=\sqrt{\hbar G / c^{3}},
$$

is the Planck length. However, in considering the classical limit of the reduced action one should include the gravitational contribution. So, for example, it is clear that also at the classical level the reduced action for a pair of "free" particles should include the Newton potential. This may be related to the above mentioned appearance of fundamental constants in the QSHJE [1][2][3]. Related to this is the Floyd observation that in the classical limit there is a residual indeterminacy depending on the integration constants [8]. Thus we see that the classical limit may in fact lead to some effect which is of quantum origin even if $\hbar$ does not appear explicitly. We also note that, in principle, the Planck constant may appear in macroscopic phenomena. This indicates that it is worth studying the structure of the quantum potential also at large scales.

It seems that the fundamental properties of $Q$ have not yet fully been investigated because the usual solutions one finds 
are essentially trivial. This is due to a clearly unsatisfactory identification, that may lead to some inconsistency, of $R e^{\frac{i}{\hbar} S_{0}}$ with the wave-function. As noticed by Floyd [8], if

$$
\operatorname{Re}^{\frac{i}{\hbar} \mathcal{S}_{0}}
$$

solves the SE, then the wave-function will in general have the form $R\left(A e^{-\frac{i}{\hbar} S_{0}}+B e^{\frac{i}{\hbar} S_{0}}\right)$. This simple remark has important consequences. So, for example, note that a real wavefunction, such as the one for bound states, simply implies $|A|=|B|$ rather than $\mathcal{S}_{0}=0$. As Einstein noticed in a letter to Bohm, the latter would imply that a quantum particle in a box is at rest and starts moving in the classical limit. Therefore, besides the mathematical consistency, identifying the wavefunction with $R e^{\frac{i}{\hbar}} S_{0}$ cannot in general lead to a quantum ana$\log$ of the reduced action. This change in the definition of $\mathcal{S}_{0}$ implies a new view of $Q$ which needs to be further investigated. In this respect we note that $Q$ provides particle's response to an external perturbation. For example, in the case of tunnelling, where according to the standard definition $\mathcal{S}_{0}$ would be vanishing, the attractive nature of $Q$ guarantees the reality of the conjugate momentum [1]. As a consequence, the role of this intrinsic energy, which is a property of all forms of matter, should manifest itself through effective interactions depending on the above fundamental constants.

It is therefore natural to consider the so called two-particle model [3], consisting of two free particles in the three dimensional space. This provides a simple physical model to investigate the structure of the interaction provided by $Q$. The QSHJE decomposes in equations for the center of mass and for the relative motion. The latter is the QSHJE

$$
\frac{1}{2 m}\left(\nabla \mathcal{S}_{0}\right)^{2}-E-\frac{\hbar^{2}}{2 m} \frac{\Delta R}{R}=0, \quad \nabla \cdot\left(R^{2} \nabla \mathcal{S}_{0}\right)=0,
$$

where

$$
r=r_{1}-r_{2}, \quad m=\frac{m_{1} m_{2}}{m_{1}+m_{2}} .
$$

Due to the quantum potential, the QSHJE has solutions in which the relative motion is not free as in the classical case. Also note that the quantum potential is negative definite. Since $\psi=\operatorname{Re}^{\frac{i}{\hbar}} \mathcal{S}_{0}$ is a solution of the SE, we have

$$
\mathcal{S}_{0}=\frac{\hbar}{2 i} \ln (\psi / \bar{\psi})
$$

so that

$$
\left(\nabla S_{0}\right)^{2}=-\frac{\hbar^{2}}{4|\psi|^{4}}(\bar{\psi} \nabla \psi-\psi \nabla \bar{\psi})^{2}
$$

where

$$
\psi=\sum_{l=0}^{\infty} \sum_{m=-l}^{l} \sum_{j=1}^{2} c_{l m j} R_{k l j}(r) Y_{l m}(\theta, \phi)
$$

with $Y_{l m}$ the spherical harmonics and $R_{k l j}$ the solutions of the radial part of the SE [3].

As $m=l \rightarrow \infty P_{l}^{l} \propto \sin ^{l} \theta$ vanishes unless $\theta=\pi / 2$, and the motion is on a plane as in the classical orbits. However, since $\lim _{l \rightarrow \infty} \partial_{\theta} P_{l}^{l}(\cos \theta)=0$, we have

$$
\lim _{m \sim l \rightarrow \infty} \partial_{\theta} P_{l}^{m}(\cos \theta)=0 .
$$

Thus, considering solutions with $c_{l m j} \neq 0$ only for sufficiently large $m$ and $l$, we have

$$
\nabla \psi=\sum_{\{l m j\}}\left(c_{l m j} R_{k l j}^{\prime} Y_{l m}, 0, \frac{i}{r} c_{l m j} R_{k l j} m Y_{l m}\right) .
$$

Depending on the coefficients $c_{l m j},\left(\nabla \mathcal{S}_{0}\right)^{2} / 2 m$ may contain nontrivial terms which do not cancel as $\hbar \rightarrow 0$. These may arise as a deformation of the classical kinetic term, which includes the centrifugal term. The $c_{l m j}$, which may depend on fundamental constants, fix the structure of the possible interaction in the two-particle model. The $c_{l m j}$ may be related to some boundary conditions implied by the geometry and the matter content of the three-dimensional space. This would relate the fundamental constants to possible collective effects [3] which may depend on cosmological aspects.

\section{ACKNOWLEDGMENTS}

I am grateful to Hans-Thomas Elze and to the sponsors of the workshop "Dice 2004" for the invitation to talk in Piombino. Work partially supported by the European Community's Human Potential Programme under contract HPRN-CT2000-00131 Quantum Spacetime and by the INTAS Research Project N.2000-254.
[1] A. E. Faraggi and M. Matone, Phys. Lett. B 450, 34 (1999); Phys. Lett. B 437, 369 (1998); Phys. Lett. A 249, 180 (1998); Phys. Lett. B 445, 77 (1998); Phys. Lett. B 445, 357 (1999); Int. J. Mod. Phys. A 15, 1869 (2000).

[2] G. Bertoldi, A. E. Faraggi and M. Matone, Class. Quant. Grav. 17, 3965 (2000).

[3] M. Matone, Found. Phys. Lett. 15, 311 (2002); hep-th/0212260.

[4] M. Matone, Int. J. Mod. Phys. A 10, 289 (1995).

[5] V. Ovsienko, "Lagrange Schwarzian derivative and symplectic
Sturm theory," CPT-93-P-2890.

[6] P. Di Francesco, P. Mathieu and D. Sénéchal, Conformal Field Theory (Springer, Berlin, 1996).

[7] A. Messiah, Quantum Mechanics, Vol. 1 (North-Holland, Amsterdam, 1961).

[8] E. R. Floyd, Phys. Rev. D25, 1547 (1982); Phys. Rev. D 26, 1339 (1982); Phys. Rev. D 29, 1842 (1984); Phys. Rev. D34, 3246 (1986); Int. J. Theor. Phys. 27, 273 (1988); Phys. Lett. A214, 259 (1996); Found. Phys. Lett. 9, 489 (1996); 
Found. Phys. Lett. 13, 235 (2000); Int. J. Mod. Phys. A 14, 1111 (1999); Int. J. Mod. Phys. A 15, 1363 (2000); quantph/0302128; quant-ph/0307090.

[9] G. Reinisch, Physica A206, 229 (1994); Phys. Rev. A56, 3409 (1997).

A. E. Faraggi, hep-th/0411118.

R. Carroll, Can. J. Phys. 77, 319 (1999); quant-ph/0309023; quant-ph/0401082; gr-qc/0406004; quant-ph/0403156; grqc/0501045.

A. Bouda, Found. Phys. Lett. 14, 17 (2001); Int. J. Mod. Phys. A 18, 3347 (2003).

A. Bouda and T. Djama, Phys. Lett. A 285, 27 (2001); Phys. Scripta 66, 97 (2002).

A. Bouda and F. Hammad, Acta Phys. Slov. 52, 101 (2002).

M. R. Brown, quant-ph/0102102.

T. Djama, quant-ph/0111121; quant-ph/0111142; quantph/0201003; quant-ph/0404098.

[10] A. E. Faraggi, hep-th/9910042.

R. Carroll, Quantum theory, deformation and integrability, North-Holland Mathematics Studies 186 (Elsevier, NorthHolland, 2000).

J. M. Delhotel, quant-ph/0401063.

R. E. Wyatt, Quantum Dynamics with trajectories, (SpringerVerlag, Berlin, 2005).

[11] M. Matone, Phys. Lett. B 357, 342 (1995); Phys. Rev. D 53 7354 (1996).

G. Bonelli and M. Matone, Phys. Rev. Lett. 76, 4107 (1996); Phys. Rev. Lett. 77, 4712 (1996).

G. Bonelli, M. Matone and M. Tonin, Phys. Rev. D 55, 6466
(1997).

M. Matone, Phys. Rev. Lett. 78, 1412 (1997).

A. E. Faraggi and M. Matone, Phys. Rev. Lett. 78, 163 (1997). R. W. Carroll, hep-th/9607219; hep-th/9610216; hepth/9702138; Nucl. Phys. B 502, 561 (1997); Lect. Notes Phys. 502, 33 (1998).

I. V. Vancea, Phys. Lett. B 480, 331 (2000); Phys. Lett. A 321, 155 (2004).

M. A. De Andrade and I. V. Vancea, Phys. Lett. B 474, 46 (2000).

M. C. B. Abdalla, A. L. Gadelha and I. V. Vancea, Phys. Lett. B 484, 362 (2000).

[12] J. Butterfield, quant-ph/0210140.

J. M. Isidro, Int. J. Mod. Phys. A 16, 3853 (2001); quantph/0105012; J. Phys. A 35, 3305 (2002); quant-ph/0112032; J. Geom. Phys. 41, 275 (2002); Phys. Lett. A 301, 210 (2002); hep-th/0204178; Mod. Phys. Lett. A 18, 1975 (2003); hepth/0304175; Mod. Phys. Lett. A 19, 349 (2004); Phys. Lett. A 317, 343 (2003); quant-ph/0310092; Mod. Phys. Lett. A 19, 1733 (2004); hep-th/0407161; Mod. Phys. Lett. A 19, 2339 (2004); quant-ph/0411166.

[13] D. M. Appleby, Found. Phys. 29, 1863 (1999); Phys. Rev. A 65, 022105 (2002); quant-ph/0308114.

B. Poirier, J. Chem. Phys. 121, 4501 (2004).

F. Girelli, E. R. Livine and D. Oriti, Nucl. Phys. B 708, 411 (2005).

M. V. John, Found. Phys. Lett. 15, 329 (2002); quant$\mathrm{ph} / 0102087$. 